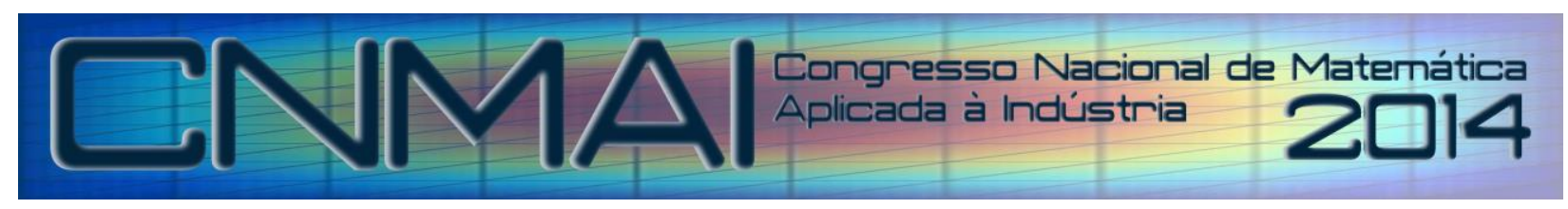

18 a 21 de novembro de 2014, Caldas Novas - Goiás

\title{
CONTROLE DE TEMPERATURA GPC/DMC COM FATOR DE PONDERAÇÃO VARIÁVEL APLICADO A UMA INCUBADORA NEONATAL
}

\author{
Felipe, C. Freitas ${ }^{1}$ \\ Francisco V. Andrade ${ }^{2}$ \\ Bismark C. Torrico ${ }^{3}$ \\ José Carlos T. Campos ${ }^{4}$ \\ ${ }^{1,3,4}$ Universidade Federal do Ceará - GPAR - DEE, Av. Mister Hull S/N Campus do Pici, Fortaleza - Ce \\ ${ }^{2}$ IFCE - Campus Cedro, Al. Dom Quintino S/N Prado, Cedro - Ce
}

\begin{abstract}
Resumo: 0 presente artigo propõe o uso do método de controle GPC com o ajuste do fator de ponderação do sinal de controle ( $\lambda$ ) variando dentro de uma faixa segundo uma função do tipo sigmóide. O método foi implementado através de simulação computacional e experimentalmente em uma incubadora neonatal para o controle de temperatura. A variação no fator de ponderação permite que se possa reduzir o valor do sobressinal bem como o valor do tempo de subida, deixando a resposta mais rápida. É feito um estudo comparativo da resposta do sistema com um fator de ponderação fixo e variável de modo a se comprovar a eficácia do método. Foram realizados ensaios também com um controlador DMC (Dynamic Matrix Control) de modo a se fazerem comparações entre os resultados com as duas técnicas de controle.
\end{abstract}

Palavras-chave: preditivo, incubadora, sigmóide, temperatura, ponderação

\section{INTRODUÇÃO}

Os recém-nascidos prematuros, ou com baixo peso, possuem limitação na capacidade de regular sua temperatura corporal, tendo uma tendência natural à hipotermia (esfriamento do corpo), o que pode levar à morte (Agostini, 2003). A produção de calor pelo corpo do recém-nascido é proveniente dos processos bioquímicos que dão sustentação a vida. A quantidade de calor produzida pelo recém-nascido necessária está entre 1.5 e $2 \mathrm{~W} / \mathrm{Kg}$ (de massa corporal). Os recémnascidos saudáveis são capazes de produzir calor adicional na faixa de 4.5 a $5 \mathrm{~W} / \mathrm{Kg}$ com o objetivo de manter uma reserva e proteção em situações de emergência como o combate a uma infecção (Costa, 2009). As principais consequências da perda de calor excessiva (hipotermia) nos seres humanos são a dificuldade de aumento/perda de massa corporal, perda de apetite, respiração irregular e até mesmo a morte. A troca de calor entre o recém-nascido e o ambiente pode ocorrer por condução, convecção, radiação e evaporação (Agostini, 2003).

Incubadoras são utilizadas no tratamento de recém-nascidos com problemas de reação a situações adversas (Uchoa et al, 2010), criando o ambiente adequado para o desenvolvimento dos mesmos. Exige-se assim, um sistema de controle eficiente de temperatura e umidade relativa do ar.

A incubadora neonatal é um equipamento médico assistencial que serve para manter a vida de recém-nascidos prematuros, por ser um ambiente termicamente neutro semelhante ao útero materno, através do controle de temperatura e umidade (Costa et al, 2009).

A incubadora neonatal proporciona ao recém-nascido um ambiente termicamente neutro que é conseguido com o controle da temperatura e da umidade relativa do ar para que permaneçam em níveis aceitáveis, mantendo, o recémnascido, a sua temperatura corporal normal a baixas taxas metabólicas, produzindo assim, o mínimo de calor possível.

A incubadora neonatal mantém o recém-nascido em um ambiente controlado sendo, portanto, favorável ao seu crescimento, ao seu desenvolvimento, à sua resistência as doenças e a sua sobrevivência. Estas incubadoras são 
construídas de acordo com a norma NBR IEC 601-2-19 que estabelece os requisitos necessários para minimizar os riscos aos pacientes e usuários, bem como sugerem testes por meio dos quais se podem avaliar o atendimento a essas exigências. Alguns artigos investigam além do controle de temperatura e umidade, a circulação de ar, luz e o nível ruído gerado pelo equipamento de acordo com a norma citada anteriormente.

Este artigo propõe o uso de um controlador GPC (Generalized Predictive Control) a ser aplicado no controle de temperatura de uma incubadora. No controlador implementado neste artigo, varia-se o valor do fator de ponderação do sinal de controle de acordo com o método do gradiente proposto em (Franca et al., 2012) para processos de primeira ordem com tempo morto. Para detalhes sobre uma análise de cuidados em ambientes hospitalares com incubadora, ver (Costa et al, 2009).

$\mathrm{O}$ uso de controladores preditivos baseados em modelos (Model Based Predictive Control) tem crescido nos últimos anos. A ideia central é o uso de um modelo que represente de maneira confiável o sistema a ser controlado e que será usado na previsão e cálculo da entrada de controle a ser utilizada. Esse tipo de controlador pode ser aplicado com bons resultados em sistemas com tempo morto. Podem ser incluídos restrições e distúrbios, sendo necessária a minimização de uma função custo, que inclui a trajetória de referência e penaliza o esforço de controle. O controlador atua usando a estratégia de horizonte deslizante, onde a saída é calculada dentro de um horizonte de previsão.

A função custo a ser minimizada inclui o valor do fator de ponderação do sinal de controle.

Este artigo está dividido da seguinte forma: A seção 2 mostra a modelagem matemática da planta a ser utilizada, a seção 3 mostra o desenvolvimento matemático do controlador GPC, a seção 4 mostra os resultados obtidos em simulação e experimental e a seção 5 finaliza com a conclusão.

\section{MODELAGEM MATEMÁTICA}

Foram realizados ensaios de identificação utilizando-se funções de primeira e de segunda ordem como modelo do sistema de controle de temperatura, sendo que para a obtenção dos resultados deste artigo foi utilizado o modelo de segunda ordem. O modelo utilizado foi obtido experimentalmente utilizando-se métodos de identificação que minimizam o erro quadrático entre a saída real e a do modelo. O teste envolveu a aplicação de uma entrada (ver Fig. 1) e foram feitas medições de temperatura. Foi utilizado um período de amostragem de 10 segundos para a obtenção dos dados de saída. A Eq. (1) mostra o modelo de segunda ordem obtido. Este modelo foi utilizado devido à sua simplicidade e a avaliação do mesmo aos dados obtidos mostrou-se aceitável. A Fig. 1 mostra a entrada aplicada. A identificação usou um método de minimização do quadrado do erro entre a saída real e do modelo.

$$
G(s)=\frac{0.179}{465.8 \mathrm{~s}^{2}+88.16 \mathrm{~s}+1} e^{-2.89 \mathrm{~s}}
$$

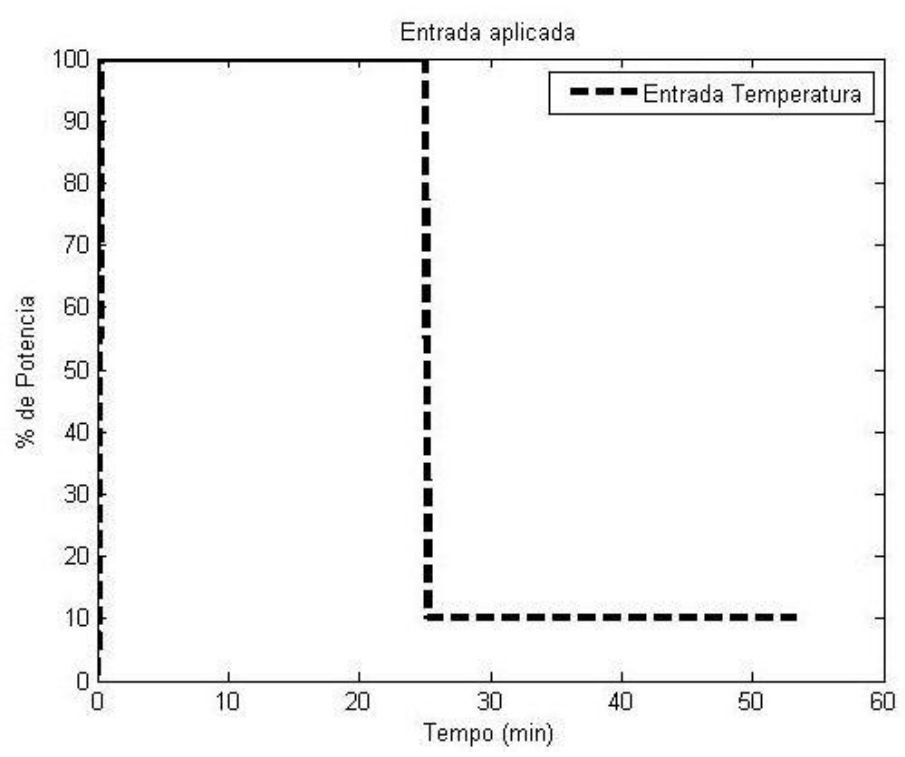

Figura 1. Entrada aplicada para identificação

A incubadora neonatal permite o controle de umidade e temperatura do interior da cúpula onde fica o recémnascido. A leitura de temperatura e umidade é feita através de um sensor SHT75 que fica localizado na parte superior da cúpula. A variação de temperatura é feita através da corrente elétrica em uma resistência. A variação de umidade é também feita através da corrente elétrica que percorre outra resistência que fica imersa em um recipiente contendo água. 
$\mathrm{O}$ ar aquecido circula entre a cúpula e a parte de baixo através de um ventilador que fica localizado na parte interna da incubadora. Existem também entradas de ar, de modo que possa ocorrer a troca do mesmo.

A Fig. 2 mostra a saída obtida e a saída do modelo obtido.

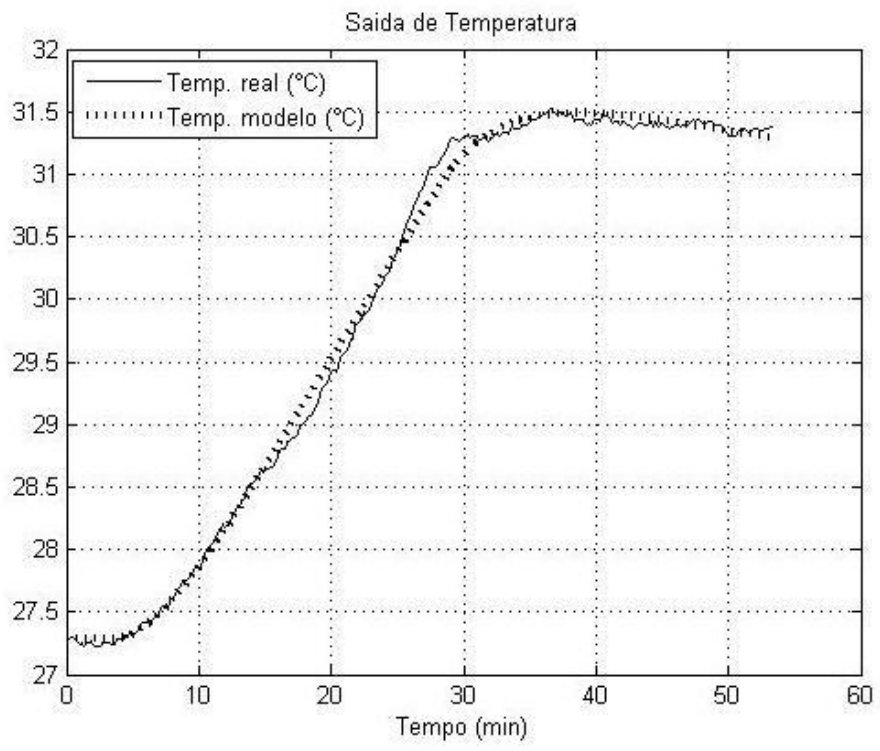

Figura 2. Saída obtida para identificação

A Fig. 3 mostra a incubadora utilizada nos experimentos. Esta incubadora pertence ao Laboratório de Automação e Robótica do Departamento de Engenharia Elétrica da Universidade Federal do Ceará.

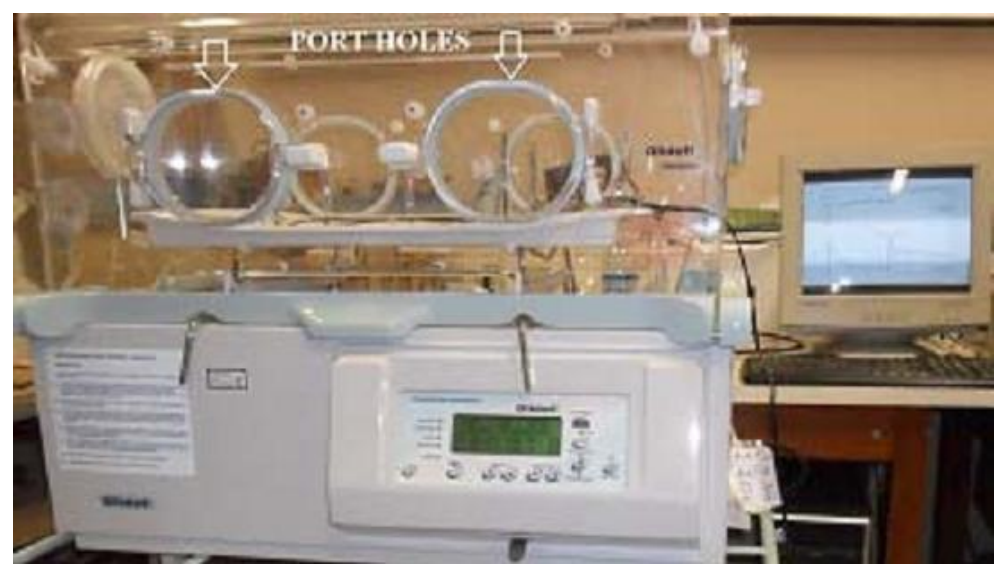

Figura 3. Incubadora Neonatal

O sistema de controle foi implementado em MATLAB® e a comunicação entre o computador e a incubadora é feito através da porta serial.

Os ensaios executados que originaram este artigo utilizaram somente a entrada de controle de temperatura, a entrada de controle de umidade foi mantida desligada durante os experimentos.

\section{MODELAGEM MATEMÁTICA}

O controle preditivo baseado em modelo (Model Predictive Control) refere-se a uma família de controladores que emprega um modelo identificado do processo para predizer o comportamento futuro sobre um horizonte de predição estendido. (Franca et al, 2012).

O método de controle preditivo baseado em modelos se refere a um conjunto de técnicas que têm em comum o uso de um modelo na obtenção do sinal de controle a ser aplicado, minimizando-se um critério de erro. Os diversos métodos diferem no uso do modelo a ser utilizado, sendo que alguns usam a resposta ao degrau, ou ao impulso, enquanto outros fazem uso do modelo de espaço de estados. O GPC faz uso da função de transferência na obtenção do modelo. É utilizado o modelo CARIMA (Controller Auto-Regressive Moving (Integraded) Average).

$\mathrm{Na}$ dedução do modelo matemático serão utilizadas as seguintes definições: 


$$
\begin{aligned}
& A\left(z^{-1}\right)=1+a_{1} z^{-1}+a_{2} z^{-1}+\ldots+a_{n} z^{-n} \\
& B\left(z^{-1}\right)=b_{0}+b_{1} z^{-1}+b_{2} z^{-1}+\ldots+a_{n} z^{-m}
\end{aligned}
$$

$y(t+j \mid t)$ é a saída prevista em $\mathrm{t}+\mathrm{j}$

$u(t)$ é a entrada de controle

O modelo CARIMA é dado pela Eq. (2):

$$
A\left(z^{-1}\right) y(t)=B\left(z^{-1}\right) u(t-1)+\frac{C\left(z^{-1}\right) e(t)}{\Delta}
$$

Onde: $\Delta=1-z^{-1}$ representa o integrador, utilizado na equação para garantir erro de regime nulo.

O método GPC, usa a equação Diophantina para obter a saída prevista e a lei de controle. A função custo a ser minimizada é dada por:

$$
J\left(N_{1}, N_{2}, N_{u}\right)=\sum_{j=N_{1}}^{N_{2}} \delta(j)[\hat{y}(t+j \mid t)-w(t+j)]^{2}+\sum_{j=1}^{N_{u}} \lambda(j)[\Delta u(t+j-1)]^{2}
$$

A saída prevista é dada por:

$$
y=G u+f
$$

Onde f é a resposta livre. Assim obtém-se após a minimização da função custo:

$$
\Delta u(t)=K(w-f)
$$

Para uma descrição mais detalhada sobre a modelagem matemática do GPC, ver (Uchoa et al, 2010).

Pode-se encontrar na literatura diversos artigos com técnicas de redução de sobressinal. O artigo de (Franca et al, 2012) propõe o método de calibração de um controlador DMC para processos de primeira ordem. No referido artigo são feitas comparações no desempenho do controlador DMC através de alguns métodos de sintonia do parâmetro $\lambda$, dentre eles: o método de Shridhar e Cooper, e de Iglesias, Sanjuan e Smith. O ajuste do fator de ponderação baseia-se no método do gradiente. Como a sintonia desse fator modifica o comportamento em malha fechada do sistema controlado (Franca et al, 2012), pode-se obter comportamentos mais ou menos agressivos com a variação desse parâmetro. Será considerado que o fator de ponderação irá variar de acordo com a Eq. (6) de uma função sigmoide:

$$
\lambda(t)=\frac{1}{1+e^{-\rho(t)}}
$$

Onde o fator $\rho(\mathrm{t})$ será atualizado pelo método do gradiente, como na Eq. (7).

$$
\rho(t)=\rho(t-1)-\mu(t) \frac{\partial J}{\partial \rho}
$$

O parâmetro $\mu(t)$ regula a estabilidade e velocidade de convergência. A otimização de $\rho$ utiliza a regra da cadeia:

$$
\begin{gathered}
\frac{\partial J}{\partial \rho}=\frac{\partial J}{\partial u} \frac{\partial u}{\partial \lambda} \frac{\partial \lambda}{\partial \rho} \\
\frac{\partial J}{\partial u}=2\left\{\left[\hat{y}(\mathrm{t}+\mathrm{j})-\mathrm{y}_{\mathrm{r}}(\mathrm{t}+\mathrm{j})\right] \mathrm{g}(\mathrm{t})+2\left[\sum_{\mathrm{j}=2}^{\mathrm{N}_{\mathrm{u}}}\left(\hat{\mathrm{y}}(\mathrm{t}+\mathrm{j})-\mathrm{y}_{\mathrm{r}}(\mathrm{t}+\mathrm{j})\right)(\mathrm{g}(\mathrm{j})-\mathrm{g}(\mathrm{j}-1))\right]\right\} \\
\frac{\partial \mathrm{u}}{\partial \lambda}=-\frac{\sum_{\mathrm{j}=1}^{\mathrm{N}_{\mathrm{y}}} \mathrm{g}(\mathrm{j}) \mathrm{e}_{0}(\mathrm{t}+\mathrm{j})}{\left(\sum_{\mathrm{j}=1}^{\mathrm{N}_{\mathrm{y}}} \mathrm{g}(\mathrm{t})^{2}+\lambda(\mathrm{t}-1)\right)^{2}} \\
\frac{\partial \lambda(t)}{\partial \rho(t)}=-\lambda(t-1)[1-\lambda(t-1)]
\end{gathered}
$$




\section{RESULTADOS}

Em todos os experimentos realizados, os parâmetros de horizonte de controle e de horizonte de predição foram mantidos fixos nos mesmos valores de $\mathrm{N}_{\mathrm{u}}=40$ (horizonte de controle) e $\mathrm{N}_{2}=80$ (horizonte de predição) de modo que se possa fazer um comparativo dos resultados com o fator de ponderação fixo e variável. Estão sendo comparados apenas os valores correspondentes à saída de temperatura. Não foram utilizadas medições na umidade.

Os valores de horizonte de controle e de predição foram sintonizados de modo que os resultados obtidos apresentassem menores valores de sobressinal.
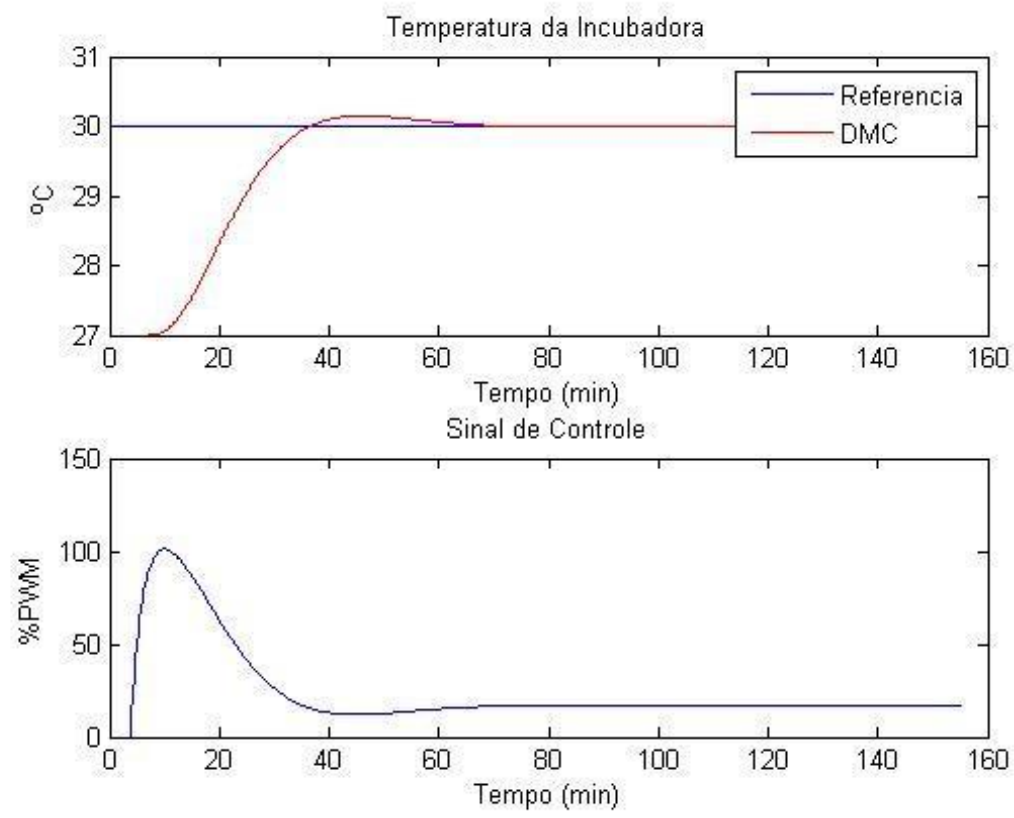

Figura 4. Simulação controle de temperatura DMC $(0.3 \leq \lambda \leq 5)$

A Fig. 4 mostra os resultados de simulação obtidos com o controlador DMC. Pode ver na Fig. 4 os sinais de saída de temperatura e do sinal de controle. Estes resultados foram obtidos com o valor do fator de ponderação variável. Foi tentado manter o sinal de controle o mais próximo possível do valor máximo sem ultrapassa-lo, de modo que se possa verificar o sobressinal e o tempo de assentamento. Neste resultado, foi utilizada uma faixa de variação do fator de ponderação $0.3 \leq \lambda \leq 5$. Pode-se verificar um sobressinal pequeno, mas o sinal segue a referência como desejado.
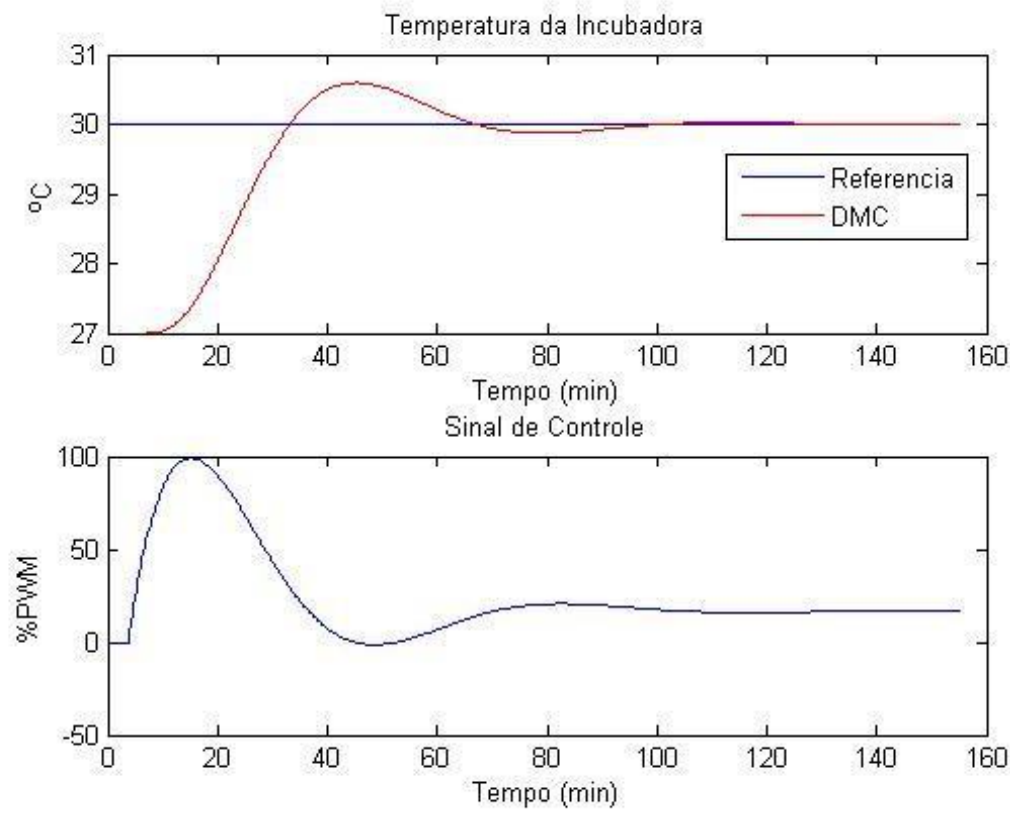

Figura 5. Simulação controle de temperatura DMC $(\lambda=0.3)$ 
A Fig. 5 mostra o sinal de controle obtido na simulação do controlador DMC com o fator de ponderação fixo em $\lambda=0.3$. Quando o valor de $\lambda$ foi aumentado, verificou-se que o esforço de controle diminuiu, entretanto houve um aumento no tempo de assentamento.
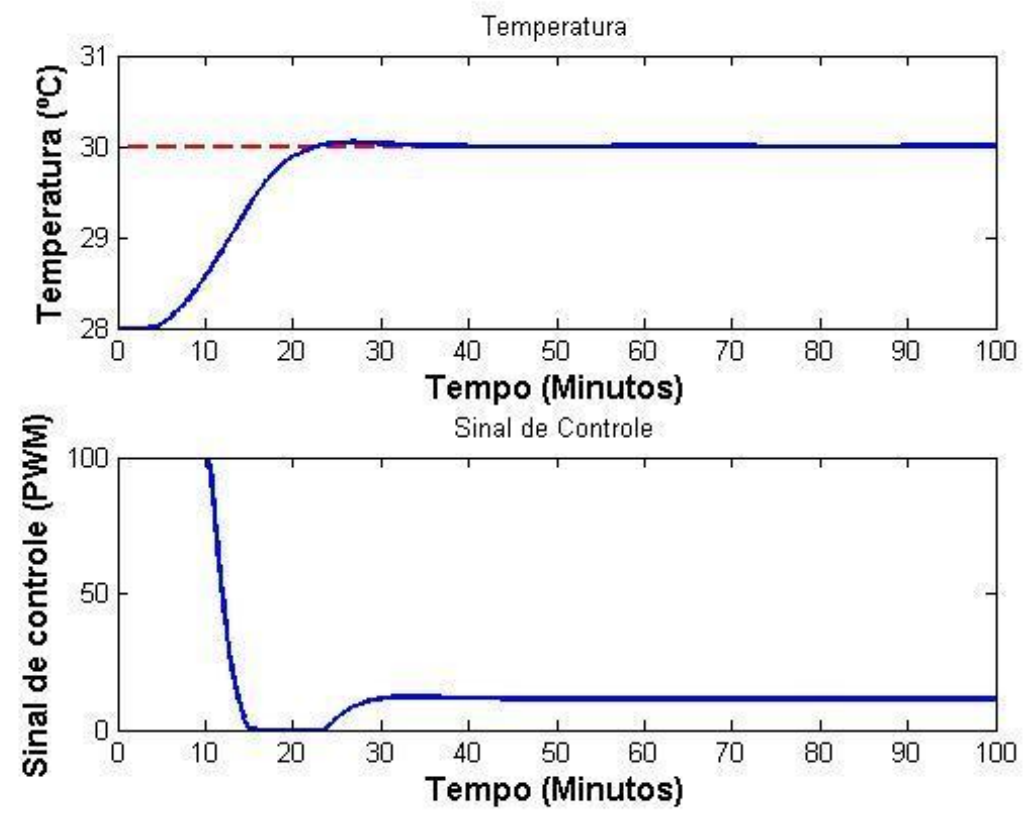

Figura 6. Simulação controle de temperatura GPC $(1 \leq \lambda \leq 2)$

A Fig. 6 mostra o resultado de simulação obtido com o controlador GPC para o fator de ponderação variando na faixa $1 \leq \lambda \leq 2$.
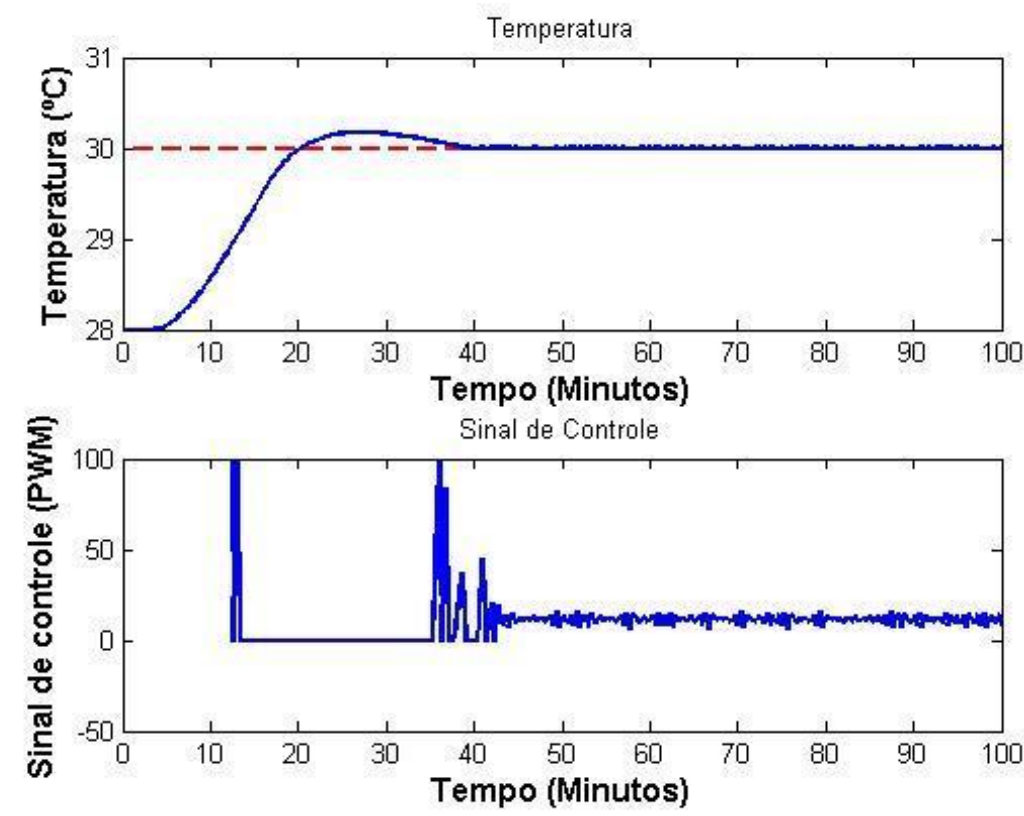

Figura 7 Simulação controle de temperatura GPC $(\lambda=2)$

Os eixos verticais nos resultados onde aparece o sinal de controle mostram o percentual de PWM aplicado à resistência de aquecimento, enquanto que o eixo horizontal mostra o tempo em minutos utilizado no ensaio. Nos resultados referentes às saídas de temperatura o eixo vertical mostra a variação em graus Celsius no interior da incubadora. 

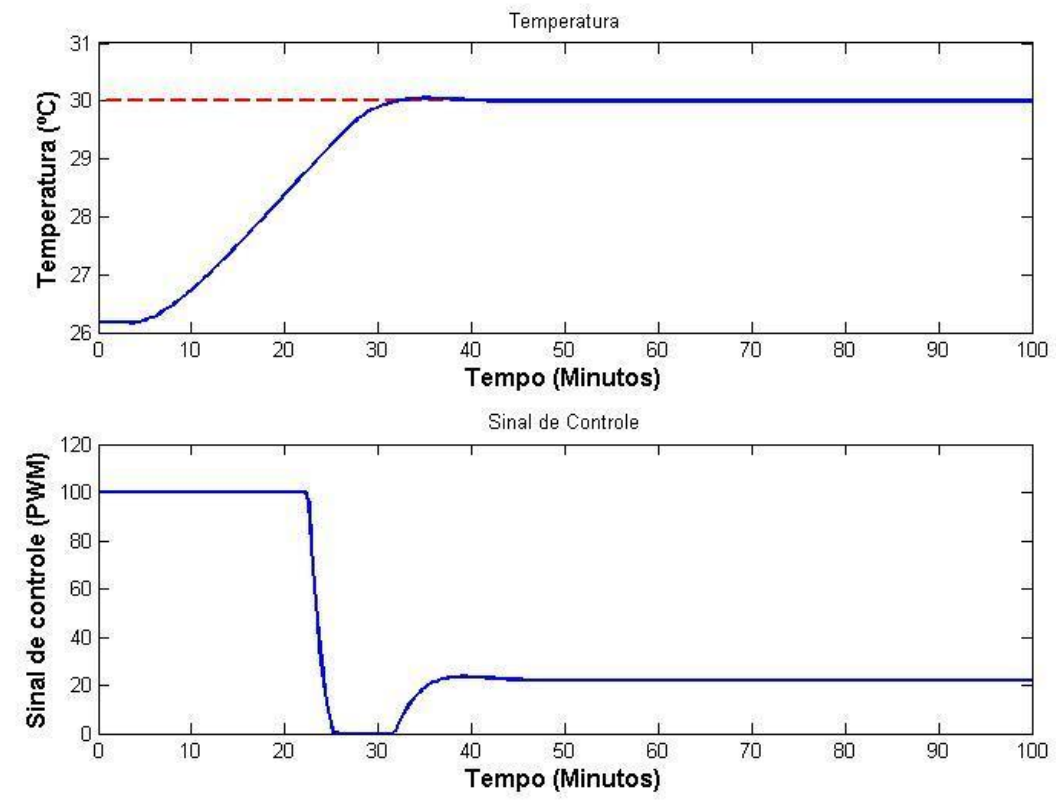

Figura 8. Saída real do controlador GPC $(1 \leq \lambda \leq 2)$

A Fig. 8 mostra um ensaio realizado com a incubadora neonatal onde o fator de ponderação foi variado entre 1 e 2 . O resultado mostrou um sinal de controle com baixo sobressinal. A resposta mostrou-se bem próxima da simulação, exceto pelo fato de que na simulação a variação é de apenas 2 graus, enquanto que o ensaio real, a variação entre a temperatura inicial e a referência foi de mais de 3 graus, o que mostra um modelo relativamente fiel à dinâmica da planta.

\section{CONCLUSÃO}

Os resultados obtidos com os valores de fator de ponderação fixos e variáveis foram satisfatórios no controle de temperatura da incubadora. Foram feitos diversos testes no sistema real, mantendo a incubadora em teste durante um período de 100 minutos. O Controlador com o fator de ponderação variável apresentou desempenho melhor que o outro controlador com fator fixo no que diz respeito a sobressinal. Foram feitos ensaios com diferentes faixas de fator de ponderação, o que em alguns casos, variou o esforço de controle, apresentando-se às vezes oscilatório. O controlador GPC foi projetado com restrições no sinal de entrada. Tentou-se realizar os ensaios de modo que as mesmas condições iniciais em todos os experimentos fossem o mais próximo possível em todos os casos, permitindo que as comparações entre os resultados ficassem facilitadas, ainda assim pode-se notar em alguns casos uma diferença entre os valores iniciais de temperatura.

É possível obter-se uma redução no sobressinal no sistema real, fazendo-se uma mudança nos valores máximo e mínimo assumidos pelo fator de ponderação. O método de variação inicia com um fator baixo, fazendo com o sinal de controle inicialmente seja elevado e a seguir este valor é aumentado segundo uma função sigmoide, reduzindo o sobressinal. $\mathrm{O}$ método utilizado permite a escolha dos valores máximo e mínimo da ponderação. Com relação às duas técnicas: DMC e GPC, o desempenho foi semelhante.

\section{AGRADECIMENTOS}

Os autores agradecem à FUNCAP e CNPq pelo suporte financeiro no desenvolvimento deste projeto de pesquisa.

\section{REFERÊNCIAS}

Agostini, N. 2003. Sistema Computadorizado para verificação da funcionalidade em Incubadoras Neonatais, Dissertação de Mestrado. Florianópolis - SC.

Brasil, Ministério da Saúde, Secretaria de Gestão de Investimentos em Saúde. Projeto REFORSUS. 2002. Equipamentos Médico-Hospitalares e o Gerenciamento da Manutenção: Capacitação à Distância, Brasília-DF.

Camacho, E. F. \& Bordons, C.1999,Model Predictive Control, Springer, Sevilla.

Cavalcante, M. U., Torrico, B. C., Neto, J. O. B., Filho, F. L. M. C., Camurça, J. L., Almeida, O. M. \& Adão, A. A. 2010. Controle de umidade e temperatura numa incubadora neonatal usando controle preditivo,XVIII Congresso Brasileiro de Automática, Bonito - MS. 
Costa, E.J.L., Freire, R.C.S., Silva, J.B.A., Cursino,C.M.P. \& Galvão, R.R.A. 2009. Uso adequado de Incubadora Neonatal na Assistência em Saúde, VIII Semestro, João Pessoa-PB.

Costa, E. J. L. 2009. Análise Crítica de Incubadoras Neonatais a partir de medições de parâmetros dos ambientes interno e externo. Tese de Doutorado, UFPB. João Pessoa - PB.

Franca, A. A., Júnior, C. A. A. L. \& Coelho, A. A. R. 2012. Calibração do Controlador DMC para Processos FOPDT, INDUSCON.

Mota, J.G.R., Albuquerque, A. A. M. \&Torrico, B. C. 2013. Controle Preditivo Generalizado Aplicado em Sistemas com Atraso de Transporte, SBAI.

Uchoa, M. C., Torrico, B.C., Neto, J.O.B., Filho, F.L.M.C., Camurça, L.J., Almeida, O.M. \& Adão, A.A. 2010. Controle de Umidade e Temperatura numa incubadora neonatal usando controle preditivo, Bonito - MG, CBA.

\title{
RESPONSABILIDADE AUTORAL
}

“Os autores são os únicos responsáveis pelo conteúdo deste trabalho”.

\section{GPC/DMC TEMPERATURE CONTROL WITH VARIABLE PONDERATION FACTOR APPLIED TO A NEONATAL INCUBATOR}

\author{
Felipe C. Freitas, camaraf12@yahoo.com.br ${ }^{1}$ \\ Francisco V. Anddrade, vanierandrade@ifce.edu.br ${ }^{2}$ \\ Bismark C. Torrico, bismark@dee.ufc.br ${ }^{3}$ \\ José Carlos T. Campos, teles@dee.ufc.br ${ }^{4}$ \\ ${ }^{1,3,4}$ Universidade Federal do Ceará - GPAR - DEE, Av. Mister Hull S/N Campus do Pici, Fortaleza - Ce \\ ${ }^{2}$ IFCE - Campus Cedro, Al. Dom Quintino S/N Prado, Cedro - Ce
}

Abstract. This article proposes using a GPC control method, by adjustment of a control signal weighting factor ( $\lambda$ ) changing in a band according a sigmoid function. This method was implemented by computational simulation and experimentally in a neo natal incubator controlling temperature. The variable weighting factor allows the reduction on overshoot as the rising time, resulting in a faster response. A comparative study for system response with and without weighting factor was done to prove the method efficiency. It was done experiments using a DMC (Dynamic Matrix Control) controller also to compare results obtained by two control techniques.

Keywords: predictive, incubator, sigmoid, temperature, ponderation 